\title{
DISCRETE APPROXIMATION OF THE POISSON-VLASOV SYSTEM*
}

BY

JACK SCHAEFFER

Carnegie-Mellon University

Abstract. Solutions of the Poisson-Vlasov system of equations that have spherical symmetry are considered. A numerical scheme which exploits the symmetry is proposed and is shown to converge in a pointwise sense. Prior convergence results due to Neunzert conclude convergence in only a weak topology although they require no symmetry.

The motion of a continuous mass (charge) distribution acting under the influence of its own gravitational (electric) field without collisions is described by the Poisson-Vlasov system of equations:

$$
\begin{gathered}
\psi_{t}+v \cdot \nabla_{x} \psi+F(x, t) \cdot \nabla_{\imath} \psi=0, \\
F(x, t)=-\nabla_{x} U(x, t), \\
U(x, t)=\gamma \int \frac{\sigma(y, t)}{|x-y|} d y, \\
\sigma(x, t)=\int \psi(x, v, t) d v .
\end{gathered}
$$

Here $\psi=\psi(x, v, t)\left(x \in \mathbf{R}^{3}, v \in \mathbf{R}^{3}, t \geqslant 0\right)$ is a scalar function describing the density in phase space, $\sigma$ is the mass (charge) density, and $F$ is the gravitational (electric) field. The constant $\gamma$ is -1 in the gravitational case and +1 in the electrical case.

The Cauchy problem is to solve (1), (2), (3), and (4) with the initial condition

$$
\psi(x, v, 0)=\dot{\psi}(x, v)
$$

for all $t>0$. Local existence of classical solutions was established by Kurth [4]. Batt [2] showed global existence in the gravitational case for spherically symmetric data (i.e., $\psi$ depends only on $|x|,|v|$, and $x \cdot v$ ). Wollman [6] extended this result to the electrical case, $\gamma=+1$. Horst [3] further extended these results to include data with cylindrical symmetry (i.e., $\dot{\psi}$ depends only on $\sqrt{x_{1}^{2}+x_{2}^{2}}, \sqrt{v_{1}^{2}+v_{2}^{2}}, x_{1} v_{1}+v_{2} v_{2}, x_{3}$, and $v_{3}$ ). Recently, Bardos 
and Degond [1] showed global existence for data that is sufficiently small in a certain norm but need not have any symmetry. The general existence problem is still open.

A natural way to generate a discrete approximation to a solution of the above Cauchy problem is to approximate the data function $\dot{\psi}$ by a large number of point masses and then follow the time evolution of the resulting many-body problem; this is called the particle-in-cell method. There is no hope for obtaining pointwise convergence for such a method since the initial error of approximating the data by point masses immediately introduces a large pointwise error. However, Neunzert [5] has established the convergence of the particle-in-cell method in a weak topology under quite general conditions. Here we consider smooth data with spherical symmetry and propose a discrete model of the continuous system, (1)-(5). In order to preserve the symmetry, the basic unit of approximation will be a spherical shell of mass (charge) rather than a point mass. Preserving the symmetry permits better estimates and this way pointwise convergence is established.

Preliminaries. We will consider only data $\dot{\psi}$ of the form $\dot{\psi}(x, v)=\grave{\phi}\left(|x|,|v|, \cos ^{-1}\left(\frac{x \cdot r}{|x||| \mid}\right)\right)$ where $\dot{\phi} \in C_{0}^{1}((0, \infty) \times(0, \infty) \times(0, \pi))$. Hence it is natural to use the variables $r=|x|$, $u=|v|$, and $\alpha=\cos ^{-1}(x \cdot v /|x||v|)$. Batt showed [2] that the resulting solution $\psi$ is continuously differentiable in $x, v$, and $t$ for all $t>0$ and that $\psi$ depends only on $r, u$, $\alpha$, and $t$ (i.e., the time evolution preserves the symmetry). We will write $\psi(x, v, t)=$ $\phi(r, u, \alpha, t)$ and note that

$$
U(x, t)=\gamma \int_{0}^{\infty} \frac{\rho(\eta, t)}{\max (r, \eta)} 4 \pi \eta^{2} d \eta
$$

and

$$
F(x, t)=\gamma M(r, t) r^{-3} x
$$

where

$$
M(r, t)=\int_{0}^{r} \rho(\eta, t) 4 \pi \eta^{2} d \eta \quad \text { and } \quad \rho(r, t)=\sigma(x, t) .
$$

Given $(r, u, \alpha) \in(0, \infty) \times(0, \infty) \times(0, \pi)$ the characteristic associated with this point is given by $z=z(r, u, \alpha, t), q=q(r, u, \alpha, t)$, and $\theta=\theta(r, u, \alpha, t)$, where

$$
\begin{aligned}
& z_{t}=q \cos \theta \text { and } z(r, u, \alpha, 0)=r, \\
& q_{t}=\gamma M(z, t) z^{-2} \cos \theta \text { and } q(r, u, \alpha, 0)=u, \\
& \theta_{t}=\left(-\gamma M(z, t) z^{-2} q^{-1}-q z^{-1}\right) \sin \theta \text { and } \theta(r, u, \alpha, 0)=\alpha .
\end{aligned}
$$

Note that $z q \sin \theta$ is independent of $t$, so we also have

$$
z_{t t}-(r u \sin \alpha)^{2} z^{-3}-\gamma M(z, t) z^{-2}=0,
$$

$z(r, u, \alpha, 0)=r$, and $z_{t}(r, u, \alpha, 0)=u \cos \alpha$.

Let $\mathscr{S}$ be the support of $\dot{\phi}$ and recall that $\mathscr{S}$ is assumed to be a compact subset of $(0, \infty) \times(0, \infty) \times(0, \pi)$. Hence there exist positive constants $R_{0}, l_{0}$, and $L_{0}$ such that if $(r, u, \alpha) \in \mathscr{S}$ then $r \leqslant R_{0}$ and $l_{0} \leqslant r u \sin \alpha \leqslant L_{0}$. A consequence of work of Batt [2] and Wollman [6] is that there exists a positive constant $U_{0}$ (depending only on $\stackrel{\phi}{\phi}$ ) such that 
$q(r, u, \alpha, t) \leqslant U_{0}$ for all $(r, u, \alpha) \in \mathscr{S}$ and $t \geqslant 0$. Hence $z(r, u, \alpha, t) \leqslant R_{0}+U_{0} t$ if $(r, u, \alpha) \in \mathscr{S}$. Also since $z q \sin \theta$ is independent of $t, l_{0} \leqslant r u \sin \alpha=z q \sin \theta \leqslant z q \leqslant z U_{0}$ so $l_{0} U_{0}^{-1} \leqslant z(r, u, \alpha, t)$ for $(r, u, \alpha) \in \mathscr{S}$ and $t \geqslant 0$. $\phi$ is constant on characteristics so

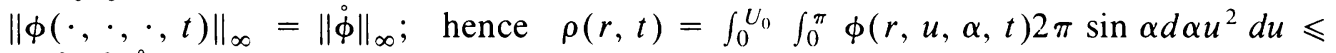
$2 \pi^{2} U_{0}^{3}\|\dot{\phi}\|_{\infty}$. To summarize, there exist positive constants $r_{0}, R_{0}, l_{0}, L_{0}, U_{0}$, and $\rho_{0}$ (depending only on $\dot{\phi}$ ) such that

$$
\begin{gathered}
r_{0} \leqslant z(r, u, \alpha, t) \leqslant R_{0}+U_{0} t, \\
l_{0} \leqslant r u \sin \alpha=z q \sin \theta \leqslant L_{0}, \\
q(r, u, \alpha, t) \leqslant U_{0}
\end{gathered}
$$

for all $(r, u, \alpha, t) \in \mathscr{S} \times[0, \infty)$ and

$$
\rho(r, t) \leqslant \rho_{0} \text { for all } r \geqslant 0 \text { and } t \geqslant 0 .
$$

The letter $C$ will denote a positive generic constant depending only on $\dot{\phi}$, and $C(t)$ will denote an increasing positive function of $t$ depending only on $\dot{\phi}$.

Another fact that will be used is that the time evolution preserves measure in $\mathbf{R}^{6}$. If the characteristics of the original system are given by $X\left(x_{0}, v_{0}, t\right)$ and $V\left(x_{0}, v_{0}, t\right)$, where $X_{t}=V, X\left(x_{0}, v_{0}, 0\right)=x_{0}$ and $V_{t}=F(X, t), V\left(x_{0}, v_{0}, 0\right)=v_{0}$, then this says

$$
\begin{aligned}
\iint & f(r, u, \alpha) \phi(r, u, \alpha, t) d v d x \\
= & \iint f\left(|x|,|v|, \cos ^{-1}\left(\frac{x \cdot v}{|x||v|}\right)\right) \psi(x, v, t) d v d x \\
& =\iint f\left(|X|,|V|, \cos ^{-1}\left(\frac{X \cdot V}{|X||V|}\right)\right) \psi\left(x_{0}, v_{0}, 0\right) d v_{0} d x_{0} \\
& =\iint f(z, q, \theta) \stackrel{\circ}{\phi}(r, u, \alpha) d v_{0} d x_{0}
\end{aligned}
$$

for all $f \in L^{\infty}((0, \infty) \times(0, \infty) \times(0, \pi))$. If $f$ is taken to be identically equal to one, we get

$$
\iint \phi(r, u, \alpha, t) d v d x=\iint \dot{\phi}(r, u, \alpha) d v_{0} d x_{0},
$$

which is mass conservation. Take $M=\iint \stackrel{\circ}{\phi}(r, u, \alpha) d v_{0} d x_{0}$ so that

$$
M=\iint \phi(r, u, \alpha, t) d v d x=\int_{0}^{\infty} \rho(r, t) 4 \pi r^{2} d r
$$

Another conserved quantity is the energy

$$
\begin{aligned}
E & =\iint \psi(x, v, t) v^{2} d v d x+\int \sigma(x, t) U(x, t) d x \\
& =\iint \phi(r, u, \alpha, t) u^{2} d v d x+\gamma \int \rho(r, t) \int_{0}^{\infty} \frac{\rho(\eta, t) 4 \pi \eta^{2}}{\max (r, \eta)} d \eta d x .
\end{aligned}
$$

Before introducing the discrete model let us consider the following continuous dependence lemma which involves only the continuous solution. 
LEMma I. There exists an increasing function $C_{1}(t)$ with $C_{1}(0) \geqslant 1$ (depending only on $\dot{\phi}$ ) such that for all $t \geqslant 0,(r, u, \alpha) \in \mathscr{S}$, and $(\eta, w, \beta) \in \mathscr{S}$

$$
\begin{aligned}
& |z(r, u, \alpha, t)-z(\eta, w, \beta, t)|+\left|z_{t}(r, u, \alpha, t)-z_{t}(\eta, w, \beta, t)\right| \\
& \quad \leqslant C_{1}(t)|(r, u, \alpha)-(\eta, w, \beta)| .
\end{aligned}
$$

This holds for $\gamma=1$ and $\gamma=-1$.

Proof. For brevity we will temporarily use the notation $z_{1}(t)=z(r, u, \alpha, t), z_{2}(t)=$ $z(\eta, w, \beta, t)$, and $Q(t)=\sup \left\{\left|z_{1}(\tau)-z_{2}(\tau)\right|+\left|z_{1}^{\prime}(\tau)-z_{2}^{\prime}(\tau)\right|: 0 \leqslant \tau \leqslant t\right\}$.

From equation (12) for $t>0$

$$
\begin{aligned}
\mid z_{1}^{\prime \prime}(t) & -z_{2}^{\prime \prime}(t)|=|(r u \sin \alpha)^{2} z_{1}^{-3}(t)+\gamma M\left(z_{1}(t), t\right) z_{1}^{-2}(t) \mid \\
\leqslant & -\left|(\eta w \sin \beta)^{2} z_{2}^{-3}(t)-\gamma M\left(z_{2}(t), t\right) z_{2}^{-2}(t)\right| \\
& +\left|M\left(z_{1}(t), t\right)-M\left(z_{2}(t), t\right)\right| z_{1}^{-2}(t)+M\left(z_{2}(t), t\right)\left|z_{1}^{-2}(t)-z_{2}^{-2}(t)\right| \\
\leqslant & (r u \sin \alpha+\eta w \sin \beta)|r u \sin \alpha-\eta w \sin \beta| r_{0}^{-3} \\
& +L_{0}^{2} z_{1}^{-3}(t) z_{2}^{-3}(t)\left|z_{2}^{3}(t)-z_{1}^{3}(t)\right|+\left|M\left(z_{1}(t), t\right)-M\left(z_{2}(t), t\right)\right| r_{0}^{-2} \\
& +M z_{1}^{-2}(t) z_{2}^{-2}(t)\left|z_{2}^{2}(t)-z_{1}^{2}(t)\right| \\
\leqslant & 2 L_{0} r_{0}^{-3}|r u \sin \alpha-\eta w \sin \beta| \\
& +L_{0} r_{0}^{-6}\left(z_{1}^{2}(t)+z_{1}(t) z_{2}(t)+z_{2}^{2}(t)\right)\left|z_{1}(t)-z_{2}(t)\right| \\
& +r_{0}^{-2}\left|M\left(z_{1}(t), t\right)-M\left(z_{2}(t), t\right)\right|+M r_{0}^{-4}\left(z_{1}(t)+z_{2}(t)\right)\left|z_{1}(t)-z_{2}(t)\right| .(21)
\end{aligned}
$$

Now note that

$|r u \sin \alpha-\eta w \sin \beta| \leqslant r u|\sin \alpha-\sin \beta|+r|u-w| \sin \beta+|r-\eta| w \sin \beta$

$$
\leqslant L_{0}|\alpha-\beta|+R_{0}|u-w|+U_{0}|r-\eta| \leqslant C|(r, u, \beta)-(\eta, w, \beta)|
$$

and

$$
\begin{aligned}
\left|M\left(z_{1}(t), t\right)-M\left(z_{2}(t), t\right)\right| & =\left|\int_{z_{1}(t)}^{z_{2}(t)} 4 \pi s^{2} \rho(s, t) d s\right| \\
& \leqslant 4 \pi\left(R_{0}+U_{0} t\right)^{2} \rho_{0}\left|z_{1}(t)-z_{2}(t)\right| .
\end{aligned}
$$

Using these in (21) we have

$$
\begin{aligned}
\mid z_{1}^{\prime \prime}(t)- & z_{2}^{\prime \prime}(t) \mid \\
\leqslant & 2 L_{0} r_{0}^{-3} C|(r, u, \alpha)-(\eta, w, \beta)|+L_{0} r_{0}^{-6} 3\left(R_{0}+U_{0} t\right)^{2}\left|z_{1}(t)-z_{2}(t)\right| \\
& +r_{0}^{-2} 4 \pi\left(R_{0}+U_{0} t\right)^{2} \rho_{0}\left|z_{1}(t)-z_{2}(t)\right|+M r_{0}^{-4} 2\left(R_{0}+U_{0} t\right)\left|z_{1}(t)-z_{2}(t)\right| \\
= & C|(r, u, \alpha)-(\eta, w, \beta)|+C(t)\left|z_{1}(t)-z_{2}(t)\right| .
\end{aligned}
$$


By (22) we have for $t \geqslant 0$

$$
\begin{aligned}
\left|z_{1}(t)-z_{2}(t)\right|+\left|z_{1}^{\prime}(t)-z_{2}^{\prime}(t)\right| & \\
\leqslant & Q(0)+\int_{0}^{t}\left(\left|z_{1}^{\prime}(\tau)-z_{2}^{\prime}(\tau)\right|+\left|z_{1}^{\prime \prime}(\tau)-z_{2}^{\prime \prime}(\tau)\right|\right) d \tau \\
\leqslant & Q(0)+\int_{0}^{t}(Q(\tau)+C|(r, u, \alpha)-(\eta, w, \beta)|+C(\tau) Q(\tau)) d \tau \\
\leqslant & |r-u|+|u \cos \alpha-w \cos \beta|+C t|(r, u, \alpha)-(\eta, w, \beta)| \\
& +(1+C(t)) \int_{0}^{t} Q(\tau) d \tau \\
& \leqslant C(t)|(r, u, \alpha)-(\eta, w, \beta)|+C(t) \int_{0}^{t} Q(\tau) d \tau .
\end{aligned}
$$

Now for $0 \leqslant \tau \leqslant t$,

$$
\left|z_{1}(\tau)-z_{2}(\tau)\right|+\left|z_{1}^{\prime}(\tau)-z_{2}^{\prime}(\tau)\right| \leqslant C(t)|(r, u, \alpha)-(\eta, w, \beta)|+C(t) \int_{0}^{t} Q(s) d s
$$

so

$$
Q(t) \leqslant C(t)|(r, u, \alpha)-(\eta, w, \beta)|+C(t) \int_{0}^{t} Q(s) d s .
$$

Since $C(t)$ is increasing we have

$$
Q(t) \leqslant C(T)|(r, u, \alpha)-(\eta, w, \beta)|+C(T) \int_{0}^{t} Q(s) d s
$$

for all $t \in[0, T]$ and $T>0$. By Gronwall's inequality

$$
Q(t) \leqslant C(T)|(r, u, \alpha)-(\eta, w, \beta)| \exp (C(T) t)
$$

for $t \in[0, T]$ so $Q(T) \leqslant C(T)|(r, u, \alpha)-(\eta, w, \beta)|$. This completes the proof of Lemma I.

The discrete model. To discretize the continuous mass distribution $\stackrel{\circ}{\phi}$ assume that $\mathscr{S}$ is the union of disjoint connected sets $S_{1}, \ldots, S_{N}$. Let

$$
\delta=\max _{i} \sup \left\{\sqrt{(r-\bar{r})^{2}+(u-\bar{u})^{2}+(\alpha-\bar{\alpha})^{2}}:(r, u, \alpha),(\bar{r}, \bar{u}, \bar{\alpha}) \in S_{i}\right\}
$$

and assume that $\delta<\min \left\{1, r_{0}\right\}$. For each $i \in\{1,2, \ldots, N\}$, choose $\left(r_{i}, u_{i}, \alpha_{i}\right) \in S_{i}$ and define $L_{i}=r_{i} u_{i} \sin \alpha_{i}, M_{i}=\iint_{S_{i}} \dot{\phi}(r, u, \alpha) d v d x$, and $z_{i}(t)=z\left(r_{i}, u_{i}, \alpha_{i}, t\right)$.

From Eq. (12) $z_{i}^{\prime \prime}(t)-L_{i}^{2} z_{i}^{-3}(t)-\gamma M\left(z_{i}(t), t\right) z_{i}^{-2}(t)=0$ with $z_{i}(0)=r_{i}$ and $z_{i}^{\prime}(0)=$ $u_{i} \cos \alpha_{i}$, so for the discrete model we define the quantities $\bar{z}_{1}(t), \bar{z}_{2}(t), \ldots, \bar{z}_{N}(t)$ (which approximate $\left.z_{1}(t), z_{2}(t), \ldots, z_{N}(t)\right)$ and $\bar{M}(r, t)$ by the system of differential equations:

$$
\bar{z}_{i}^{\prime \prime}-L_{i}^{2}\left(\bar{z}_{i}\right)^{-3}-\gamma \bar{M}\left(\bar{z}_{i}, t\right)\left(\bar{z}_{i}\right)^{-2}=0
$$

with $\bar{z}_{i}(0)=r_{i}$ and $\bar{z}_{i}^{\prime}(0)=u_{i} \cos \alpha_{i}$ for $i=1,2, \ldots, N$ and

$$
\bar{M}(r, t)=\sum_{i=1}^{N} M_{i} \xi\left(r-\bar{z}_{i}(t)\right) \text {, }
$$


where we define $\xi: \mathbf{R} \rightarrow \mathbf{R}$ by

$$
\xi(r)= \begin{cases}0 & \text { if } r \leqslant 0, \\ r \delta^{-1} & \text { if } 0<r<\delta, \\ 1 & \text { if } \delta \leqslant r .\end{cases}
$$

$\bar{M}(r, t)$ is Lipschitz continuous in $r$ for $r \geqslant 0$ and $r_{0} \leqslant \bar{z}_{i}(0) \leqslant R_{0}$ for each $i$ so there exists $T>0$ such that a unique solution to the system (23) and (24) exists for $0 \leqslant t<T$ and satisfies

$$
\frac{1}{2} r_{0} \leqslant \bar{z}_{i}(t) \leqslant 2\left(R_{0}+U_{0} t\right)
$$

for each $i$ and for $0 \leqslant t<T$. We will take $T$ to be the maximal such time and note that $T$ is heavily dependent on the discretization; however it will be shown later that $T$ tends to infinity as $\delta$ tends to zero.

TheOrem I. Let $\stackrel{\circ}{\phi} \in C_{0}^{1}((0, \infty) \times(0, \infty) \times(0, \pi)), z(r, u, \alpha, t)$ as in (9), (10), and (11), $M(r, t)$ as in (8), and $\bar{z}_{i}(t), \bar{M}(r, t)$ as above. There exists a positive increasing function $C_{2}(t)$ (which is dependent only on $\dot{\phi}$ and not on $\delta$ ) such that for all $i \in\{1,2, \ldots, N\}$, $(r, u, \alpha) \in S_{i}$, and $t \in[0, T)$ :

$$
\left|z(r, u, \alpha, t)-\bar{z}_{i}(t)\right|+\left|z_{t}(r, u, \alpha, t)-\bar{z}_{i}^{\prime}(t)\right| \leqslant C_{2}(t) \delta
$$

and

$$
|M(\eta, t)-\bar{M}(\eta, t)| \leqslant C_{2}(t) \delta \text { for all } \eta \geqslant 0 .
$$

This holds for $\gamma=1$ and $\gamma=-1$.

Proof. Define

$$
\overline{\bar{M}}(r, t)=\sum_{i=1}^{N} M_{i} \xi\left(r-z_{i}(t)\right)
$$

for $r \geqslant 0$ and $t \geqslant 0$. The proof is divided into parts $\mathrm{A}, \mathrm{B}$, and C. Part A will estimate $M-\overline{\bar{M}}$ and part B will estimate $\overline{\bar{M}}-\bar{M}$.

Part A. Fix $t>0$ and $(\eta, w, \beta) \in \mathscr{S}$. Choose $i$ so that $(\eta, w, \beta) \in S_{i}$ and define $I=\{j$ : $\left.z_{j}(t) \leqslant z(\eta, w, \beta, t)\right\}$. Note that if $(r, u, \alpha) \in S_{j}$, then by Lemma I $\left|z(r, u, \alpha, t)-z_{j}(t)\right| \leqslant$ $C_{1}(t) \delta$. Hence, if $(r, u, \alpha) \in S_{j}$ and $j \in I$,

$$
z(r, u, \alpha, t) \leqslant z_{j}(t)+C_{1}(t) \delta \leqslant z(\eta, w, \beta, t)+C_{1}(t) \delta,
$$

that is, if $j \in I$, then

$$
S_{j} \subset\left\{(r, u, \alpha): z(r, u, \alpha, t) \leqslant z(\eta, w, \beta, t)+C_{1}(t) \delta\right\}
$$

and hence

$$
\bigcup_{j \in I} S_{j} \subset\left\{(r, u, \alpha): z(r, u, \alpha, t) \leqslant z(\eta, w, \beta, t)+C_{1}(t) \delta\right\} .
$$

Now denoting $z=z(\eta, w, \beta, t)$,

$$
\overline{\bar{M}}(z, t)=\sum_{j=1}^{N} M_{j} \xi\left(z-z_{j}(t)\right) \leqslant \sum_{j \in I} M_{j}
$$


since $j \notin I$ implies $z-z_{j}(t) \leqslant 0$ which implies $\xi\left(z-z_{j}(t)\right)=0$ by (25). Also by (30)

$$
\begin{aligned}
\sum_{j \in I} M_{j} & =\iint_{\cup_{j \in I} S_{j}} \dot{\phi}(r, u, \alpha) d v d x \\
& \leqslant \iint_{\left\{(r, u, \alpha): z(r, u, \alpha, t) \leqslant z+C_{1}(t) \delta\right\}} \stackrel{\circ}{\phi}(r, u, \alpha) d v d x \\
& =\iint_{\left\{r \leqslant z+C_{1}(t) \delta\right\}} \phi(r, u, \alpha, t) d v d x=M\left(z+C_{1}(t) \delta, t\right) \\
& =M(z, t)+\int_{z}^{z+C_{1}(t) \delta} 4 \pi s^{2} \rho(s, t) d s \leqslant M(z, t)+C(t) \delta .
\end{aligned}
$$

Therefore

$$
\overline{\bar{M}}(z, t) \leqslant M(z, t)+C(t) \delta .
$$

To bound $\overline{\bar{M}}$ from below define $J=\left\{j: z_{j}(t) \leqslant z-C_{1}(t) \delta\right\}$, where $z=z(\eta, w, \beta, t)$ as before. If $(r, u, \alpha) \in S_{j}$ and $z(r, u, \alpha, t) \leqslant z-2 C_{1}(t) \delta$, then by Lemma I, $z_{j}(t) \leqslant$ $z(r, u, \alpha, t)+C_{1}(t) \delta \leqslant z-C_{1}(t) \delta$, that is $j \in J$. Therefore,

$$
\mathscr{S} \cap\left\{(r, u, \alpha): z(r, u, \alpha, t) \leqslant z-2 C_{1}(t) \delta\right\} \subset \bigcup_{j \in J} S_{j}
$$

If $j \in J$ then $z_{j}(t) \leqslant z-C_{1}(t) \delta \leqslant z-\delta$, so $z-z_{j}(t) \geqslant \delta$, and hence $\xi\left(z-z_{j}(t)\right)=1$. Therefore

$$
\begin{aligned}
\overline{\bar{M}}(z, t)= & \sum_{j=1}^{N} M_{j} \xi\left(z-z_{j}(t)\right) \geqslant \sum_{j \in J} M_{j}=\iint_{\cup_{j \in J} S_{j}} \stackrel{\AA}{\phi}(r, u, \alpha) d v d x \\
\geqslant & \int_{\mathscr{S} \cap\left\{(r, u, \alpha): z(r, u, \alpha, t) \leqslant z-2 C_{1}(t) \delta\right\}} \dot{\phi}(r, u, \alpha) d v d x \\
= & \int_{\left\{(r, u, \alpha): z(r, u, \alpha, t) \leqslant z-2 C_{1}(t) \delta\right\}} \grave{\phi}(r, u, \alpha) d v d x \\
= & \iint_{\left\{r \leqslant z-2 C_{1}(t) \delta\right\}} \phi(r, u, \alpha, t) d v d x \\
= & M\left(\max \left\{0, z-2 C_{1}(t) \delta\right\}, t\right) \\
= & M(z, t)-\int_{\max \left\{0, z-2 C_{1}(t) \delta\right\}}^{z} 4 \pi s^{2} \rho(s, t) d s \\
\geqslant & M(z, t)-C(t) \delta .
\end{aligned}
$$

Combining this and (31) we have

$$
|M(z, t)-\overline{\bar{M}}(z, t)| \leqslant C(t) \delta
$$

for $z=z(\eta, w, \beta, t)$ and $(\eta, w, \beta) \in \mathscr{S}$.

We will need (32) for all $z$, not just for $z \in z(\mathscr{S}, t)$. Note that if $r_{1} \geqslant R_{0}+U_{0} t+\delta$ then $M\left(r_{1}, t\right)=\overline{\bar{M}}\left(r_{1}, t\right)=M$, and if $0 \leqslant r_{1} \leqslant r_{0}$ then $M\left(r_{1}, t\right)=\overline{\bar{M}}\left(r_{1}, t\right)=0$. Hence we consider $t \geqslant 0$ and $r_{1} \in\left[r_{0}, R_{0}+U_{0} t+1\right]$, such that $r_{1} \notin z(\mathscr{S}, t)$. Define $r_{2}=\inf \{\eta$ : $\eta \geqslant r_{0}$ and $\left.\left(\eta, r_{1}\right) \cap z(\mathscr{S}, t)=\varnothing\right\}$ and note that $r_{0} \leqslant r_{2} \leqslant r_{1}$ and $\left(r_{2}, r_{1}\right) \cap z(\mathscr{S}, t)=\varnothing$. 
We have

$$
\begin{aligned}
M\left(r_{1}, t\right)-M\left(r_{2}, t\right) & =\int_{r_{2}}^{r_{1}} 4 \pi s^{2} \rho(s, t) d s \\
& =\int_{\left\{(x, v):|x| \in\left(r_{2}, r_{1}\right)\right\}} \phi(r, u, \alpha, t) d v d x \\
& =\int_{\left\{(\eta, w, \beta): z(\eta, w, \beta, t) \in\left(r_{2}, r_{1}\right)\right\}} \stackrel{\AA}{\phi}(\eta, w, \beta) d v d x \\
& =0 \text { since }\left\{(\eta, w, \beta): z(\eta, w, \beta, t) \in\left(r_{2}, r_{1}\right)\right\} \cap \mathscr{S}
\end{aligned}
$$

is empty.

$z_{j}(t) \notin\left(r_{2}, r_{1}\right)$ for all $j$ so by $(25)$

$$
\begin{aligned}
\overline{\bar{M}}\left(r_{1}, t\right)-\overline{\bar{M}}\left(r_{2}, t\right) & =\sum_{j=1}^{N} M_{j}\left(\xi\left(r_{1}-z_{j}(t)\right)-\xi\left(r_{2}-z_{j}(t)\right)\right) \\
& \leqslant \sum_{\left\{j: z_{j}(t) \in\left(r_{2}-\delta, r_{2}\right]\right\}} M_{j} .
\end{aligned}
$$

If $z_{j}(t) \in\left(r_{2}-\delta, r_{2}\right]$ then by Lemma I

$$
\begin{aligned}
z\left(S_{j}, t\right) & \subset\left[z_{j}(t)-C_{1}(t) \delta, z_{j}(t)+C_{1}(t) \delta\right] \\
& \subset\left[r_{2}-\delta-C_{1}(t) \delta, r_{2}+C_{1}(t) \delta\right]
\end{aligned}
$$

so

$$
\begin{aligned}
0 \leqslant & \overline{\bar{M}}\left(r_{1}, t\right)-\overline{\bar{M}}\left(r_{2}, t\right) \\
& \leqslant \quad \int_{\substack{\cup_{j} \\
j: z_{j}(t) \in\left(r_{2}-\delta, r_{2}\right]}} \dot{\phi}(r, u, \alpha) d v d x \\
& \leqslant \quad \iint_{\left\{(r, u, \alpha):\left|z(r, u, \alpha, t)-r_{2}\right| \leqslant \delta+C_{1}(t) \delta\right\}} \stackrel{\phi}{\phi}(r, u, \alpha) d v d x \\
& =\int_{\left\{(x, v):|| x\left|-r_{2}\right| \leqslant \delta+C_{1}(t) \delta\right\}} \phi(r, u, \alpha, t) d v d x \\
& =\int_{\max \left\{0, r_{2}-\delta-C_{1}(t) \delta\right\}}^{r_{2}+\delta+C_{1}(t) \delta} 4 \pi s^{2} \rho(s, t) d s \\
\leqslant & 4 \pi\left(r_{2}+1+C_{1}(t)\right)^{2} \rho_{0} 2\left(1+C_{1}(t)\right) \delta \\
\leqslant & C(t) \delta, \text { since } r_{2} \leqslant R_{0}+U_{0} t+1 .
\end{aligned}
$$

Recall that by definition $r_{2}=\inf \left\{\eta: \eta \geqslant r_{0}\right.$ and $\left.\left(\eta, r_{1}\right) \cap z(\mathscr{S}, t)=\varnothing\right\}$. The map $(r, u, \alpha) \rightarrow z(r, u, \alpha, t)$ is continuous by Lemma $\mathrm{I}$ and $\mathscr{S}$ is compact; hence $z(\mathscr{S}, t)$ is compact. If $r_{2}>r_{0}$ then $r_{2} \in z(\mathscr{S}, t)$ and so by (32)

$$
\left|M\left(r_{2}, t\right)-\overline{\bar{M}}\left(r_{2}, t\right)\right| \leqslant C(t) \delta .
$$


If $r_{2}=r_{0}$ then $M\left(r_{2}, t\right)=\overline{\bar{M}}\left(r_{2}, t\right)=0$ so (35) still holds. Now combining (33), (34), and (35) we have

$$
\begin{aligned}
& \left|M\left(r_{1}, t\right)-\overline{\bar{M}}\left(r_{1}, t\right)\right| \\
& \quad \leqslant\left|M\left(r_{1}, t\right)-M\left(r_{2}, t\right)\right|+\left|M\left(r_{2}, t\right)-\overline{\bar{M}}\left(r_{2}, t\right)\right|+\left|\overline{\bar{M}}\left(r_{2}, t\right)-\overline{\bar{M}}\left(r_{1}, t\right)\right| \\
& \quad \leqslant 0+C(t) \delta+C(t) \delta .
\end{aligned}
$$

Therefore

$$
|M(r, t)-\overline{\bar{M}}(r, t)| \leqslant C(t) \delta \quad \text { for all } r \geqslant 0 \text { and } t \geqslant 0
$$

Part B. In order to estimate $\bar{M}-\overline{\bar{M}}$ let us define for $0 \leqslant t<T$

$$
\|z-\bar{z}\|(t)=\sup _{0 \leqslant \tau \leqslant t} \max _{1 \leqslant i \leqslant N}\left|z_{i}(\tau)-\bar{z}_{i}(\tau)\right| .
$$

Let $r \geqslant 0$ and $t \in[0, T)$. If $z_{j}(t) \geqslant r+\|z-\bar{z}\|(t)$,

$$
r-z_{j}(t) \leqslant-\|z-\bar{z}\|(t) \leqslant 0,
$$

and

$$
r-\bar{z}_{j}(t) \leqslant r-\left(z_{j}(t)-\|z-\bar{z}\|(t)\right) \leqslant 0
$$

so

$$
\xi\left(r-z_{j}(t)\right)=\xi\left(r-\bar{z}_{j}(t)\right)=0 .
$$

Similarly, if $z_{j}(t) \leqslant r-\|z-\bar{z}\|(t)-\delta$, then

$$
r-z_{j}(t) \geqslant\|z-\bar{z}\|(t)+\delta \geqslant \delta
$$

and

$$
r-\bar{z}_{j}(t) \geqslant r-\left(z_{j}(t)+\|z-\bar{z}\|(t)\right) \geqslant \delta,
$$

so

$$
\xi\left(r-z_{j}(t)\right)=\xi\left(r-\bar{z}_{j}(t)\right)=1 .
$$

Hence

$$
\begin{aligned}
|\bar{M}(r, t)-\overline{\bar{M}}(r, t)| & =\left|\sum_{j=1}^{N} M_{j}\left(\xi\left(r-\bar{z}_{j}(t)\right)-\xi\left(r-z_{j}(t)\right)\right)\right| \\
& \leqslant \sum_{j \in K} M_{j}
\end{aligned}
$$

where

$$
K=\left\{j:\left|r-z_{j}(t)\right|<\|z-\bar{z}\|(t)+\delta\right\} .
$$


Now if $j \in K$ and $(\eta, w, \beta) \in S_{j}$ then

$$
\left|z_{j}(t)-r\right|<\|z-\bar{z}\|(t)+\delta
$$

and by Lemma I

$$
\left|z(\eta, w, \beta, t)-z_{j}(t)\right| \leqslant C_{1}(t) \delta
$$

so

$$
|z(\eta, w, \beta, t)-r|<\|z-\bar{z}\|(t)+\delta+C_{1}(t) \delta
$$

Therefore

$$
\bigcup_{j \in K} S_{j} \subset\{(\eta, w, \beta):|z(\eta, w, \beta, t)-r|<\varepsilon\}
$$

where $\varepsilon=\|z-\bar{z}\|(t)+\delta+C_{1}(t) \delta$. Now by (38) we have

$$
\begin{aligned}
|\bar{M}(r, t)-\overline{\bar{M}}(r, t)| & \leqslant \sum_{j \in K} M_{j} \\
& =\iint_{\cup_{j \in K} S_{j}} \AA(\eta, w, \beta) d v d x \\
& \leqslant \int_{\{(\eta, w, \beta):|z(\eta, w, \beta, t)-r|<\varepsilon\}} \stackrel{\circ}{\phi}(\eta, w, \beta) d v d x \\
& =\int_{\{(x, v):|| x|-r|<\varepsilon\}} \psi(x, v, t) d v d x \\
& =\int_{\max \{0, r-\varepsilon\}}^{r+\varepsilon} 4 \pi s^{2} \rho(s, t) d s \leqslant 4 \pi(r+\varepsilon)^{2} \rho_{0} 2 \varepsilon .
\end{aligned}
$$

Recall that $\varepsilon=\| z-\bar{z}||(t)+\delta+C_{1}(t) \delta$. Now $\left|z_{j}(\tau)-\bar{z}_{j}(\tau)\right| \leqslant\left|z_{j}(\tau)\right|+\left|\bar{z}_{j}(\tau)\right| \leqslant 3\left(R_{0}\right.$ $\left.+U_{0} t\right)$ for all $\tau \in[0, t]$ so $\|z-\bar{z}\|(t) \leqslant 3\left(R_{0}+U_{0} t\right)$ and $\varepsilon \leqslant 3\left(R_{0}+U_{0} t\right)+1+C_{1}(t)$. If $r \leqslant 2 R_{0}+2 U_{0} t+1$ we have by (39),

$$
\begin{aligned}
|\bar{M}(r, t)-\overline{\bar{M}}(r, t)| & \leqslant 4 \pi\left(5\left(R_{0}+U_{0} t\right)+2+C_{1}(t)\right)^{2} \rho_{0} 2 \varepsilon \\
& =C(t) \varepsilon=C(t)\left(\|z-\bar{z}\|(t)+\delta+C_{1}(t) \delta\right) \\
& =C(t)\|z-\bar{z}\|(t)+C(t) \delta .
\end{aligned}
$$

If $r>2 R_{0}+2 U_{0} t+1$, then $\bar{M}(r, t)=\overline{\bar{M}}(r, t)=M$ so for all $r \geqslant 0$ and $t \in[0, T)$

$$
|\bar{M}(r, t)-\overline{\bar{M}}(r, t)| \leqslant C(t)\|z-\bar{z}\|(t)+C(t) \delta .
$$

Combining (40) and (36) we get for $r \geqslant 0$ and $t \in[0, T$ )

$$
\begin{aligned}
|M(r, t)-\bar{M}(r, t)| & \leqslant|M(r, t)-\overline{\bar{M}}(r, t)|+|\overline{\bar{M}}(r, t)-\bar{M}(r, t)| \\
& \leqslant C(t) \delta+C(t)\|z-\bar{z}\|(t)+C(t) \delta \\
& =C(t) \delta+C(t)\|z-\bar{z}\|(t) .
\end{aligned}
$$


Part C. In this section we use (41) and the differential equations for $z_{i}$ and $\bar{z}_{i}$ to estimate $\|z-\bar{z}\|(t)$. From Eqs. (12) and (23)

$$
\begin{aligned}
\left|z_{i}^{\prime \prime}(t)-\bar{z}_{i}^{\prime \prime}(t)\right|= & \left|L_{i}^{2} z_{i}^{-3}+\gamma M\left(z_{i}, t\right) z_{i}^{-2}-L_{i}^{2}\left(\bar{z}_{i}\right)^{-3}-\gamma \bar{M}\left(\bar{z}_{i}, t\right)\left(\bar{z}_{i}\right)^{-2}\right| \\
\leqslant & L_{i}^{2}\left|z_{i}^{-3}-\left(\bar{z}_{i}\right)^{-3}\right|+M\left(z_{i}, t\right)\left|z_{i}^{-2}-\left(\bar{z}_{i}\right)^{-2}\right| \\
& +\left(\bar{z}_{i}\right)^{-2}\left|M\left(z_{i}, t\right)-\bar{M}\left(\bar{z}_{i}, t\right)\right| \\
\leqslant & L_{0}^{2} z_{i}^{-3}\left(\bar{z}_{i}\right)^{-3}\left|z_{i}^{3}-\left(\bar{z}_{i}\right)^{3}\right|+M z_{i}^{-2}\left(\bar{z}_{i}\right)^{-2}\left|z_{i}^{2}-\left(\bar{z}_{i}\right)^{2}\right| \\
& +\left(\bar{z}_{i}\right)^{-2}\left(\left|M\left(z_{i}, t\right)-M\left(\bar{z}_{i}, t\right)\right|+\left|M\left(\bar{z}_{i}, t\right)-\bar{M}\left(\bar{z}_{i}, t\right)\right|\right)
\end{aligned}
$$

for $0 \leqslant t<T$. Note that

$$
\begin{aligned}
\left|M\left(z_{i}, t\right)-M\left(\bar{z}_{i}, t\right)\right| & =\left|\int_{\bar{z}_{i}}^{z_{i}} 4 \pi s^{2} \rho(s, t) d s\right| \\
& \leqslant 4 \pi\left(\max \left\{z_{i}, \bar{z}_{i}\right\}\right)^{2} \rho_{0}\left|z_{i}-\bar{z}_{i}\right| \leqslant C(t)\|z-\bar{z}\|(t)
\end{aligned}
$$

and by $(41)$

$$
\left|M\left(\bar{z}_{i}, t\right)-\bar{M}\left(\bar{z}_{i}, t\right)\right| \leqslant C(t) \delta+C(t)\|z-\bar{z}\|(t)
$$

so

$$
\left|M\left(z_{i}, t\right)-M\left(\bar{z}_{i}, t\right)\right|+\left|M\left(\bar{z}_{i}, t\right)-\bar{M}\left(\bar{z}_{i}, t\right)\right| \leqslant C(t) \delta+C(t)\|z-\bar{z}\|(t) .
$$

Using this in (42) yields for $0 \leqslant t<T$

$$
\begin{aligned}
\mid z_{i}^{\prime \prime}(t)- & \bar{z}_{i}^{\prime \prime}(t) \mid \\
\leqslant & L_{0}^{2} z_{i}^{-3}\left(\bar{z}_{i}\right)^{-3}\left(z_{i}^{2}+z_{i}\left(\bar{z}_{i}\right)+\left(\bar{z}_{i}\right)^{2}\right)\left|z_{i}-\bar{z}_{i}\right| \\
& +M z_{i}^{-2}\left(\bar{z}_{i}\right)^{-2}\left(z_{i}+\bar{z}_{i}\right)\left|z_{i}-\bar{z}_{i}\right|+\left(\bar{z}_{i}\right)^{-2}(C(t) \delta+C(t)\|z-\bar{z}\|(t)) \\
\leqslant & L_{0}^{2} r_{0}^{-3}\left(\frac{1}{2} r_{0}\right)^{-3} 7\left(R_{0}+U_{0} t\right)^{2}\|z-\bar{z}\|(t) \\
& +M r_{0}^{-2}\left(\frac{1}{2} r_{0}\right)^{-2} 3\left(R_{0}+U_{0} t\right)\|z-\bar{z}\|(t)+\left(\frac{1}{2} r_{0}\right)^{-2}(C(t) \delta+C(t)\|z-\bar{z}\|(t)) \\
= & C(t) \delta+C(t)\|z-\bar{z}\|(t) .
\end{aligned}
$$

Define for $0 \leqslant t<T$

$$
Q(t)=\sup _{0 \leqslant \tau \leqslant t} \max _{1 \leqslant i \leqslant N}\left(\left|z_{i}(\tau)-\bar{z}_{i}(\tau)\right|+\left|z_{i}^{\prime}(\tau)-\bar{z}_{i}^{\prime}(\tau)\right|\right) .
$$


Note that $z_{i}(0)=\bar{z}_{i}(0)=r_{i}$ and $z_{i}^{\prime}(0)=\bar{z}_{i}^{\prime}(0)=u_{i} \cos \alpha_{i}$, so by $(43)$

$$
\begin{aligned}
\left|z_{i}(t)-\bar{z}_{i}(t)\right|+\left|z_{i}^{\prime}(t)-\bar{z}_{i}^{\prime}(t)\right| & \leqslant \int_{0}^{t}\left(\left|z_{i}^{\prime}(\tau)-\bar{z}_{i}^{\prime}(\tau)\right|+\left|z_{i}^{\prime \prime}(\tau)-\bar{z}_{i}^{\prime \prime}(\tau)\right|\right) d \tau \\
& \leqslant \int_{0}^{t}(Q(\tau)+C(\tau) \delta+C(\tau) Q(\tau)) d \tau \\
& \leqslant C(t) \delta+C(t) \int_{0}^{t} Q(\tau) d \tau .
\end{aligned}
$$

Hence for all $\tau \in[0, t]$ and $i \in\{1,2, \ldots, N\}$,

$$
\begin{aligned}
\left|z_{i}(\tau)-\bar{z}_{i}(\tau)\right|+\left|z_{i}^{\prime}(\tau)-\bar{z}_{i}^{\prime}(\tau)\right| & \leqslant C(\tau) \delta+C(\tau) \int_{0}^{\tau} Q(s) d s \\
& \leqslant C(t) \delta+C(t) \int_{0}^{t} Q(s) d s
\end{aligned}
$$

and therefore $Q(t) \leqslant C(t) \delta+C(t) \int_{0}^{t} Q(s) d s$ for $0 \leqslant t<T$. For $\tau \in[0, t]$ and $t<T$, $Q(\tau) \leqslant C(t) \delta+C(t) \int_{0}^{\tau} Q(s) d s$ since $C(t)$ is increasing. By Gronwall's inequality we get for $0 \leqslant \tau \leqslant t, Q(\tau) \leqslant C(t) \delta \exp (C(t) \tau)$ so

$$
Q(t) \leqslant C(t) \delta \text { for } 0 \leqslant t<T .
$$

Finally by (44) and Lemma I if $(r, u, \alpha) \in S_{i}$ and $0 \leqslant t<T$ then

$$
\begin{aligned}
\left|z(r, u, \alpha, t)-\bar{z}_{i}(t)\right|+\mid z_{t}(r, u, \alpha, t)- & \bar{z}_{i}^{\prime}(t) \mid \\
\leqslant & \left|z(r, u, \alpha, t)-z_{i}(t)\right|+\left|z_{i}(t)-\bar{z}_{i}(t)\right| \\
& +\left|z_{t}(r, u, \alpha, t)-z_{i}^{\prime}(t)\right|+\left|z_{i}^{\prime}(t)-\bar{z}_{i}^{\prime}(t)\right| \\
\leqslant & C_{1}(t) \delta+C(t) \delta=C(t) \delta .
\end{aligned}
$$

Also by (41) and (44) for $0 \leqslant r$ and $0 \leqslant t<T$

$$
\begin{aligned}
|M(r, t)-\bar{M}(r, t)| & \leqslant C(t) \delta+C(t)\|z-\bar{z}\|(t) \\
& \leqslant C(t) \delta+C(t) C(t) \delta=C(t) \delta .
\end{aligned}
$$

This completes the proof of Theorem I.

Comment. Recall that the existence time $T$ depends on $\delta$. We can now use Theorem I to show that $T$ tends to infinity as $\delta$ tends to zero: Let $T_{0}>0$ and $\delta \in\left(0, r_{0} /\left(3 C_{2}\left(T_{0}\right)\right)\right)$. Then for all $j$ and for $0 \leqslant t<\min \left(T, T_{0}\right)$ we have by (27)

$$
\begin{aligned}
\frac{2}{3} r_{0} & \leqslant r_{0}-C_{2}\left(T_{0}\right) \delta \leqslant r_{0}-C_{2}(t) \delta \leqslant z_{j}(t)-\left|\bar{z}_{j}(t)-z_{j}(t)\right| \\
& \leqslant \bar{z}_{j}(t) \leqslant z_{j}(t)+\left|\bar{z}_{j}(t)-z_{j}(t)\right| \\
& \leqslant R_{0}+U_{0} t+C_{2}(t) \delta \leqslant R_{0}+U_{0} t+\frac{1}{3} r_{0}<\frac{3}{2}\left(R_{0}-U_{0} t\right) .
\end{aligned}
$$

Now if $T \leqslant T_{0}$ the solution $\bar{z}_{1}, \bar{z}_{2}, \ldots, \bar{z}_{N}$ can be continued beyond $t=T$, which is a contradiction. Therefore $T>T_{0}$. 
Recall from (19) that the energy is conserved; we may write

$$
\begin{aligned}
E= & \iint \phi(r, u, \alpha, t) u^{2} d v d x+\gamma \int \rho(r, t) \int_{0}^{\infty} \frac{\rho(\eta, t)}{\max (r, \eta)} 4 \pi \eta^{2} d \eta d x \\
= & \iint \phi(r, u, \alpha, t)\left(u^{2}+\gamma \int_{0}^{\infty} \frac{\rho(\eta, t)}{\max (r, \eta)} 4 \pi \eta^{2} d \eta\right) d v d x \\
= & \iint \phi(r, u, \alpha, t)\left(u^{2}+\gamma \iint \frac{\phi\left(r_{0}, u_{0}, \alpha_{0}, t\right)}{\max \left(r, r_{0}\right)} d v_{0} d x_{0}\right) d v d x \\
= & \iint \dot{\phi}(r, u, \alpha)\left(q^{2}(r, u, \alpha, t)\right) \\
& +\gamma \iint \frac{\stackrel{\circ}{\phi}\left(r_{0}, u_{0}, \alpha_{0}\right) d v_{0} d x_{0}}{\max \left(z(r, u, \alpha, t), z\left(r_{0}, u_{0}, \alpha_{0}, t\right)\right)} d v d x .
\end{aligned}
$$

Also since

$$
z_{t}(r, u, \alpha, t)=q(r, u, \alpha, t) \cos (\theta(r, u, \alpha, t))
$$

and

$$
\begin{aligned}
& z(r, u, \alpha, t) q(r, u, \alpha, t) \sin (\theta(r, u, \alpha, t))=r u \sin \alpha \\
q^{2}(r, u, \alpha, t)= & q^{2}(r, u, \alpha, t) \cos ^{2}(\theta(r, u, \alpha, t))+q^{2}(r, u, \alpha, t) \sin ^{2}(\theta(r, u, \alpha, t)) \\
= & z_{t}^{2}(r, u, \alpha, t)+(r u \sin \alpha)^{2} z^{-2}(r, u, \alpha, t)
\end{aligned}
$$

Substituting (46) into (45) yields

$$
\begin{aligned}
E= & \iint \dot{\phi}(r, u, \alpha)\left(z_{t}^{2}(r, u, \alpha, t)\right. \\
& \left.+\frac{(r u \sin \alpha)^{2}}{z^{2}(r, u, \alpha, t)}+\gamma \iint \frac{\stackrel{\circ}{\phi}\left(r_{0}, u_{0}, \alpha_{0}\right) d v_{0} d x_{0}}{\max \left(z(r, u, \alpha, t), z\left(r_{0}, u_{0}, \alpha_{0}, t\right)\right)}\right) d v d x
\end{aligned}
$$

(47) suggests a quantity which will be nearly conserved by the discrete system.

COROllary I. There exists a positive increasing function $C_{3}(t)$ (depending only on $\stackrel{\circ}{\phi}$ ) such that for all $t \in[0, T)$

$$
\left|E-\sum_{i=1}^{N} M_{i}\left(\left(\bar{z}_{i}^{\prime}(t)\right)^{2}+L_{i}^{2}\left(\bar{z}_{i}(t)\right)^{-2}+\sum_{j=1}^{N} \frac{\gamma M_{j}}{\max \left(\bar{z}_{i}(t), \bar{z}_{j}(t)\right)}\right)\right| \leqslant C_{3}(t) \delta .
$$

Proof. For brevity denote $z=z(r, u, \alpha, t)$ and $z_{0}=z\left(r_{0}, u_{0}, \alpha_{0}, t\right)$. Note that

$$
\begin{aligned}
\left|\frac{1}{\max \left(z, z_{0}\right)}-\frac{1}{\max \left(\bar{z}_{i}(t), \bar{z}_{j}(t)\right)}\right| & \leqslant\left|\frac{1}{\max \left(z, z_{0}\right)}-\frac{1}{\max \left(\bar{z}_{i}(t), z_{0}\right)}\right| \\
& +\left|\frac{1}{\max \left(\bar{z}_{i}(t), z_{0}\right)}-\frac{1}{\max \left(\bar{z}_{i}(t), \bar{z}_{j}(t)\right)}\right| \\
& \leqslant \frac{\left|z-\bar{z}_{i}(t)\right|}{\left(\frac{1}{2} r_{0}\right)^{2}}+\frac{\left|z_{0}-\bar{z}_{j}(t)\right|}{\left(\frac{1}{2} r_{0}\right)^{2}} .
\end{aligned}
$$


If $(r, u, \alpha) \in S_{i}$ and $\left(r_{0}, u_{0}, \alpha_{0}\right) \in S_{j}$, then by Theorem I and (49)

$$
\left|\frac{1}{\max \left(z, z_{0}\right)}-\frac{1}{\max \left(\bar{z}_{i}(t), \bar{z}_{j}(t)\right)}\right| \leqslant \frac{C_{2}(t) \delta}{\left(r_{0} / 2\right)^{2}}+\frac{C_{2}(t) \delta}{\left(r_{0} / 2\right)^{2}}=\frac{8 C_{2}(t) \delta}{r_{0}^{2}} .
$$

Therefore if $(r, u, \alpha) \in S_{i}$, then

$$
\begin{aligned}
\left|\iint \frac{\grave{\phi}\left(r_{0}, u_{0}, \alpha_{0}\right)}{\max \left(z, z_{0}\right)} d v_{0} d x_{0}-\sum_{j=1}^{N} \frac{M_{j}}{\max \left(\bar{z}_{i}(t), \bar{z}_{j}(t)\right)}\right| \\
=\left|\sum_{j=1}^{N}\left(\iint_{S_{j}} \frac{\stackrel{\phi}{\phi}\left(r_{0}, u_{0}, \alpha_{0}\right)}{\max \left(z, z_{0}\right)} d v_{0} d x_{0}-\frac{M_{j}}{\max \left(\bar{z}_{i}(t), \bar{z}_{j}(t)\right)}\right)\right| \\
=\left|\sum_{j=1}^{N} \iint_{S_{j}} \dot{\phi}\left(r_{0}, u_{0}, \alpha_{0}\right)\left(\frac{1}{\max \left(z, z_{0}\right)}-\frac{1}{\max \left(\bar{z}_{i}(t), \bar{z}_{j}(t)\right)}\right) d v_{0} d x_{0}\right| \\
\leqslant \sum_{j=1}^{N} \iint_{S_{j}} \dot{\phi}\left(r_{0}, u_{0}, \alpha_{0}\right) \frac{8 C_{2}(t) \delta}{r_{0}^{2}} d v_{0} d x_{0}=8 M r_{0}^{-2} C_{2}(t) \delta=C(t) \delta .
\end{aligned}
$$

Again for $(r, u, \alpha) \in S_{i}$ we have

$$
\begin{aligned}
\mid\left(z_{t}^{2}+\frac{(r u \sin \alpha)^{2}}{z^{2}}\right) & -\left(\left(\bar{z}_{i}^{\prime}(t)\right)^{2}+\frac{L_{i}^{2}}{\left(\bar{z}_{i}(t)\right)^{2}}\right) \mid \\
\leqslant & \left|z_{t}^{2}-\left(\bar{z}_{i}^{\prime}(t)\right)^{2}\right|+z^{-2}\left|(r u \sin \alpha)^{2}-L_{i}^{2}\right|+L_{i}^{2}\left|z^{-2}-\left(\bar{z}_{i}(t)\right)^{-2}\right| \\
\leqslant & \left|z_{t}+\bar{z}_{i}^{\prime}(t)\right|\left|z_{t}-\bar{z}_{i}^{\prime}(t)\right|+r_{0}^{-2}\left(r u \sin \alpha+L_{i}\right)\left|r u \sin \alpha-L_{i}\right| \\
& +L_{0}^{2} z^{-2}\left(\bar{z}_{i}(t)\right)^{-2}\left(\bar{z}_{i}(t)+z\right)\left|\bar{z}_{i}(t)-z\right| .
\end{aligned}
$$

By Theorem I, $\left|z_{t}-\bar{z}_{i}^{\prime}(t)\right| \leqslant C_{2}(t) \delta$ so

$$
\left|z_{t}+\bar{z}_{i}^{\prime}(t)\right| \leqslant\left|2 z_{t}\right|+\left|\bar{z}_{i}^{\prime}(t)-z_{t}\right| \leqslant 2 U_{0}+C_{2}(t) \delta
$$

and

$$
\begin{aligned}
& \left|z_{t}^{2}+\frac{(r u \sin \alpha)^{2}}{z^{2}}-\left(\left(\bar{z}_{i}^{\prime}(t)\right)^{2}+\frac{L_{i}^{2}}{\left(\bar{z}_{i}(t)\right)^{2}}\right)\right| \\
& \quad \leqslant\left(2 U_{0}+C_{2}(t) \delta\right) C_{2}(t) \delta+r_{0}^{-2} 2 L_{0} C \delta+L_{0}^{2} r_{0}^{-2}\left(\frac{1}{2} r_{0}\right)^{-2} 3\left(R_{0}+U_{0} t\right) C_{2}(t) \delta \\
& \quad \leqslant C(t) \delta
\end{aligned}
$$


Now to finish the proof we rewrite $E$ as in (47) and use the bounds given by (50) and (51):

$$
\begin{aligned}
\mid E & -\sum_{i=1}^{N} M_{i}\left(\left(\bar{z}_{i}^{\prime}(t)\right)^{2}+L_{i}^{2}\left(\bar{z}_{i}(t)\right)^{-2}+\sum_{j=1}^{N} \frac{\gamma M_{j}}{\max \left(\bar{z}_{i}(t), \bar{z}_{j}(t)\right)}\right) \mid \\
= & \mid \sum_{i=1}^{N} \iint_{S_{i}} \dot{\phi}(r, u, \alpha)\left(z_{t}^{2}+\frac{(r u \sin \alpha)^{2}}{z^{2}}+\gamma \iint \frac{\grave{\phi}\left(r_{0}, u_{0}, \alpha_{0}\right)}{\max \left(z, z_{0}\right)} d v_{0} d x_{0}\right. \\
& -\left(\bar{z}_{i}^{\prime}(t)\right)^{2}-L_{i}^{2}\left(\bar{z}_{i}(t)\right)^{-2}-\gamma \sum_{j=1}^{N} \frac{M_{j}}{\max \left(\bar{z}_{i}(t), \bar{z}_{j}(t)\right)} d v d x \mid \\
\leqslant & \sum_{i=1}^{N} \iint_{S_{i}} \dot{\phi}(r, u, \alpha)\left|z_{t}^{2}+\frac{(r u \sin \alpha)^{2}}{z^{2}}-\left(\bar{z}_{i}^{\prime}(t)\right)^{2}-\frac{L_{i}^{2}}{\left(\bar{z}_{i}(t)\right)^{2}}\right| \\
& \left.+\left|\int_{j} \frac{\dot{\phi}\left(r_{0}, u_{0}, \alpha_{0}\right)}{\max \left(z, z_{0}\right)} d v_{0} d x_{0}-\sum_{j=1}^{N} \frac{M_{j}}{\max \left(\bar{z}_{i}(t), \bar{z}_{j}(t)\right)}\right|\right) d v d x \\
\leqslant & \sum_{i=1}^{N} \iint_{S_{i}} \dot{\phi}(r, u, \alpha)(C(t) \delta+C(t) \delta) d v d x \\
= & 2 M C(t) \delta .
\end{aligned}
$$

This completes the proof of Corollary I.

\section{REFERENCES}

[1] C. Bardos and P. Degond, Global existence for the Vlasov-Poisson equation in 3 space variables with small initial data. Preprint.

[2] J. Batt, Global symmetric solutions of the initial value problem of stellar dynamics, J. Differential Equations 25 , 342-364 (1977)

[3] E. Horst, On the classical solutions of the initial value problem for the unmodified nonlinear Vlasov equation, Parts 1 and 2, Math. Methods Appl. Sci. 3, 229-248 (1981); 4, 19-32 (1982)

[4] R. Kurth, Das Anfangswertproblem der Stellardynamik, Z. Astrophys. 30, 213-229 (1952)

[5] H. Neunzert, Mathematical investigations on particle-in-cell methods, Fluid Dynamics Trans. 9, 229-254 (1978)

[6] S. Wollman, The spherically symmetric Vlasov-Poisson system, J. Differential Equations 35, 30-35 (1980) 